\title{
Corela
}

Cognition, représentation, langage

HS-8 | 2010

L'interpellation

\section{Formes et fonctions de l'interpellation en situation didactique}

\section{Claire Doquet-Lacoste}

\section{OpenEdition}

\section{Journals}

Édition électronique

URL : http://journals.openedition.org/corela/1731

DOI : 10.4000/corela.1731

ISSN : $1638-573 \mathrm{X}$

\section{Éditeur}

Cercle linguistique du Centre et de I'Ouest - CerLICO

Référence électronique

Claire Doquet-Lacoste, «Formes et fonctions de l'interpellation en situation didactique », Corela [En ligne], HS-8 | 2010, mis en ligne le 24 novembre 2010, consulté le 03 mai 2019. URL : http:// journals.openedition.org/corela/1731; DOI : 10.4000/corela.1731

Ce document a été généré automatiquement le 3 mai 2019.

\section{(c) (i) (2)(2)}

Corela - cognition, représentation, langage est mis à disposition selon les termes de la licence Creative Commons Attribution - Pas d'Utilisation Commerciale - Partage dans les Mêmes Conditions 4.0 International. 


\title{
Formes et fonctions de l'interpellation en situation didactique
}

\author{
Claire Doquet-Lacoste
}

1 L'interpellation est une opération langagière polymorphe et, de ce fait, difficile à classer linguistiquement. Elle n'est pas répertoriée dans les dictionnaires de sciences du langage habituels, ce qui laisse la liberté aux auteurs de décider ce que recouvre le terme, selon le paradigme dans lequel ils évoluent. Interpeller, ce peut être apostropher (Détrie 2007), émettre une interjection (Charaudeau 1992), nommer (Noailly, 1995), topicaliser (Berthoud, 1996)... sans bien sûr que ces actions langagières soient exclusives les unes des autres. Dès lors se pose un problème de délimitation du champ : qu'est-ce qui relève spécifiquement de l'interpellation? Qu'est qui est à en exclure, et pourquoi ? Dans cette question se joue en particulier l'articulation entre la nomination et l'utilisation des interjections, qui font apparaître deux fonctions conjointes dans le terme même d'interpellation: l'appellation et la mise en relation entre deux personnes. C'est dans cette perspective que je voudrais évoquer les formes d'interpellations que l'on peut observer en situation de classe.

2 Je m'attacherai à expliciter l'intimité du lien entre interpellation et situation didactique. Il me semble en effet que l'interpellation est intrinsèquement liée au didactique, au sens large incluant l'acception actuelle ( «la » didactique) et l'acception rhétorique de « genre destiné à instruire ». En effet, dans tous les discours didactiques, la relation établie avec l'auditeur ou le lecteur paraît aussi importante, si ce n'est plus, que la fonction référentielle du discours. Aujourd'hui, c'est bien en termes de relation et d'interaction que les spécialistes situent l'action éducative (Develay 2007, Sensevy \& Mercier 2008).

Dans le cadre de ce projet large, explorer des corpus didactiques variés pour y repérer des constantes interpellatives, j'ai choisi de m'intéresser prioritairement aux formes de l'interpellation dans un type de corpus bien plus restreint: la situation d'interaction maitre-élève en classe. C'est-à-dire le type même de situation didactique contemporaine, observée du double point de vue de la classe en temps réel, donc des interactions orales, 
et des interactions moins visibles mais tenaces que constituent les commentaires des enseignants sur les copies des élèves. C'est un exemple d'interpellations à l'écrit, dans la marge du devoir de l'élève, qui permet un autre regard sur cet acte de langage qu'est l'interpellation.

Le matériau que j'ai exploré m'a conduite à me poser des questions de trois ordre :

- D'abord, ce qui concerne le statut de l'interpellation en situation didactique : du point de vue des actes de langage, comment peut se décrire l'interpellation en contexte de classe? comment s'insère-t-elle dans l'activité principale du maître, l'enseignement ? quelle place at-elle dans le langage magistral, à côté d'autres énoncés plus clairement didactiques au sens où ils sont porteurs de contenus d'apprentissage?

- Ensuite, ce qui concerne les formes de l'interpellation en classe : quels types de lexèmes sont-ils utilisés, et par qui ? Plus largement, comment caractériser et classer les séquences interpellatives? Ce questionnement va poser le problème des limites de l'interpellation, selon que l'on se situe dans une appréhension pragmatique du phénomène ou que l'on s'en tienne à la syntaxe. Le classement des formes va nous aider, je pense, à y voir plus clair sur les différentes dimensions de l'interpellation. Nous ferons là un parallèle entre formes orales et formes écrites.

- Enfin, ce qui concerne les fonctions de l'interpellation en classe : qui interpelle qui, et comment ? À cette première question, dont les spécificités de la situation didactique, un maitre et des élèves, rendent non évidente la réponse, s'ajoute la question du « pourquoi » : qu'est-ce qui motive les interpellations, en particulier celles du maitre ? Le fonctionnement de l'interpellation, presque uniquement sur le modus alors que le discours enseignant se fonde sur le dictum (ce qui est à enseigner), paraît en partie ancré dans la rythmique de la profération.

\section{Interpeller, est-ce nommer? Remarques préliminaires à vocation définitoire sur l'interpellation.}

5 Le verbe interpeller est attesté par le Robert Historique de la langue française au sens de « réclamer, invoquer » en 1364, puis chez Rabelais, « au sens étymologique de couper la parole à quelqu'un (1534) (le latin interpellare signifie "interrompre quelqu'un qui parle»). Cette signification m'intéresse, particulièrement dans le contexte didactique : c'est souvent dans ce sens premier que les élèves interpellent l'enseignant, par la parole ou par le geste, et de fait ils interrompent le discours magistral. Quant aux circonstances dans lesquelles un enseignant interpelle un élève, deux configurations sont très répandues :

- dans le cas d'un cours de type frontal, où la parole est principalement dévolue à

l'enseignant, l'interpellation intervient le plus souvent pour couper court à une conversation engagée avec un camarade : il s'agit donc de l'interruption de quelqu'un qui parle;

- dans le cas d'un cours de type dialogal, où la parole est répartie entre l'enseignant et les élèves, c'est en général l'enseignant qui la distribue, et dans ce cas l'interpellation est une incitation à parler ; mais même dans cette configuration, il s'agit bien pour l'interpellant de couper court à une activité préalable de l'interpellé et de le faire entrer dans une autre activité (prise de parole ou pas).

6 L'interpellation serait donc synonyme d'interruption d'une parole par une autre, ou plus largement interruption d'une activité par une prise de parole. Il faut à présent 
caractériser linguistiquement cette parole interruptive pour tenter de délimiter le champ interpellatif.

$7 \mathrm{Si}$, comme il en ressort des travaux sus-cités, l'interpellation peut consister en une apostrophe (nominalisante) ou une interjection, les deux catégories devraient être substituables l'une à l'autre dans les énoncés interpellatifs. Je me propose ici de partir d'un corpus d'énoncés intuitivement reconnus (par des observateurs extérieurs et non linguistes) comme des interpellations, pour examiner les cas où interjection et apostrophe peuvent, ou non, se substituer l'une à l'autre et commuter.

Dans les exemples examinés ici, seront toujours donnés :

- l'énoncé attesté en contexte didactique et reconnu comme interpellation (numéroté a),

- le résultat de commutations et de substitutions, non attestés mais également reconnus comme interpellations (numérotés $b, c$, $d$, etc).

9 Exemple 1 : interpellation dans un énoncé assertif.

(1a) (attesté) Bon- on y va - les consignes sont au tableau je perds pas de temps

(1b) Les enfants- on y va - les consignes sont au tableau je perds pas de temps

(1c) Bon alors- on y va - les consignes sont au tableau je perds pas de temps

(1d) Bon- les enfants- on y va - les consignes sont au tableau je perds pas de temps

Interjection ou nom propre en apostrophe, les éléments interpellatifs paraissent substituables l'un à l'autre. Ils peuvent également être juxtaposés dans les cas suivants :

- interjection + interjection (interjection + adverbe ?') (1c)

- interjection $+\mathrm{GN}$.

11 En revanche, la disposition GN + interjection est rejetée :

(1e) * Les enfants alors- on y va - les consignes sont au tableau je perds pas de temps

12 Exemple 2 : interpellation dans un énoncé injonctif :
(2a) Damien - arrête ça
(2b) Hé - arrête ça
(2c) Damien - au travail
(2d) Hé - au travail

13 En revanche, la postposition au prédicat est possible pour le GN mais pas pour l'interjection, qui est syntaxiquement contrainte en début de phrase :

(2e) Au travail- Damien

(2f) * Au travail- hé ${ }^{2}$

14 Cette limite des possibilités de substitution et de commutation des GN et interjections, constatée dans les deux exemples développés ici, ${ }^{3}$ permet de délimiter plus strictement l'interpellation: si celle-ci n'advient que lorsque la substitution $\mathrm{GN} /$ interjection est possible, est-il légitime de parler d'interpellation dans le cas d'une apostrophe nominale ne se trouvant pas en tête de phrase ? Catherine Détrie s'est posé la question, y compris dans le présent volume, et elle estime que l'apostrophe par Npropre n'est pas proprement interpellative mais doit plutôt être considérée comme un terme d'adresse, où la fonction de saillance est bien plus importante que l'interpellation. D'un point de vue énonciatif, considérer que l'interpellation se trouve nécessairement en tête de phrase, comme préliminaire au topic (Schegloff, 1980, cité par Berthoud, 1996), revient à dire qu'elle réside avant tout dans la rupture du fil énonciatif, comme le montre l'exemple 3 (extrait d'un entretien avec une enseignante évoquant une séance en classe, auto-confrontation) : 
(3a) là je me suis dit - hé ho- ça va pas- tu dérailles complètement - et c'est pour ça que j'ai réorienté après- ils ne comprenaient rien en fait

(3b) là je me suis dit - Agnès- ça va pas- tu dérailles complètement - et c'est pour ça que j'ai réorienté après- ils ne comprenaient rien en fait

Interjection ou apostrophe nominale, l'interpellation - qui est ici une auto-interpellation en discours rapporté - marque la rupture entre la narration et le DR.

Exemple 4 : énoncés formés d'un GN ou d'une interjection.

GN et interjection sont substituables lorsqu'ils forment à eux seuls un énoncé exclamatif :

Damien! / Hé!

En revanche, ils ne paraissent pas interchangeables dans l'échange suivant :

(4a) M : comment s'appelait le deuxième petit cochon

$\mathrm{E}:$ (lève le doigt)

$\mathrm{M}:$ Kevin

E : Nif-Nif

(4b) M : comment s'appelait le deuxième petit cochon

$\mathrm{E}$ : (lève le doigt)

$\mathrm{M}:{ }^{*} \mathrm{Hé}$

E : Nif-Nif

La rupture énonciative (le maitre s'adresse à toute la classe / à un élève précisément) a donc l'air ici de pouvoir être marquée par un GN mais pas par une interjection, ce qui met en question les propos tenus précédemment et identifiant interpellation et rupture. Toutefois, en se demandant où réside la rupture dans ce contexte discursif, on voit qu'elle est sans doute moins dans les propos du maître que dans le geste de l'élève qui, en levant le doigt, focalise l'attention du maitre et transforme l'échange entre maître et groupe classe en un échange entre maître et individu élève. L'apostrophe survient après que l'interpellé se soit désigné comme parleur potentiel. Par conséquent, l'association de l'interpellation et de la rupture énonciative n'est pas invalidée. Il faudra se demander nous le ferons par la suite - dans quelle mesure un geste (en l'occurrence l'autodésignation par lever du doigt) constitue ou pas une interpellation.

20 Le critère syntaxique de substitution / commutation permet donc de caractériser l'interpellation comme un acte allocutif qui crée une rupture énonciative. En ce sens, l'interpellation est donc l'acte inaugural du changement de niveau énonciatif. C'est sur cette caractérisation que je m'appuierai pour étudier diverses manifestations de l'interpellation en situation didactique.

$\mathrm{Au}$ caractère hybride reconnu à l'interpellation fait écho son statut, également hybride, en situation didactique. En effet, l'interpellation y constitue tout à la fois :

- Le seul acte de langage laissé au libre choix de l'élève : ce dernier est libre d'interpeller ou non le maître, au moment qu'il choisit. Toutes les autres prises de parole en classe sont contraintes par la demande du maître (qui demande à un élève ou à des élèves de répondre, ou de venir au tableau exposer leur travail, par exemple. Et qui pour ce faire interpelle les élèves).

- Un acte de langage qui échappe à l'enseignement, au sens où on n'enseigne pas l'interpellation. Ce qui n'empêche pas le maître de la contrôler, par exemple de corriger un élève qui va l'interpeller par « hé ! », ou bien de mettre en cause l'opportunité d'une interpellation («c'est pour ça que tu m'interromps?»).

- Un acte de langage qui, de la part de celui qui l'accomplit, échappe en partie au contrôle méta-énonciatif. Prenons l'exemple de l'enseignement des langues étrangères à l'école. Une 
classe de CM2, un cours d'anglais. Les élèves se sont attribué des prénoms anglais, c'est avec ces prénoms qu'ils s'apostrophent. Le maître est en train de parler anglais, tout à coup un élève tombe de sa chaise. Eclat de rire général, et le maître se met en colère : « Marin! Qu'est-ce que tu fais encore ! ». L'interpellation apparaît comme l'espace discursif de l'expressivité et de l'émotion, la part la plus « contrôlée » de l'énonciation laisse place à davantage de spontanéité, et dans le cas exposé ce moindre contrôle est clairement marqué par l'utilisation de la langue maternelle.

\section{Les formes de l'interpellation en situation de classe} forme scolaire ritualisée : le geste de lever la main que peut faire un élève qui va, de fait, interrompre le discours de l'enseignant. Peut-on parler d'interpellation? Peut-être, si l'on considère les facteurs suivants :

- Il y a bien une interruption de la parole magistrale.

- Cette interruption est délibérée : c'est ce qui donne au lever du doigt un statut de signe, conventionnel et décodable, alors que par exemple un élève qui tombe de sa chaise provoquera la même interruption de la parole du maître, et sans doute des réactions fortes des élèves, sans que le tomber de chaise soit pour autant un geste, et encore moins un signe, interpellatif.

- À ce signe interpellatif, le maitre répond comme il répondrait à une interpellation orale : « Oui, X ? » « Qu'est-ce qu'il y a, X ? » « Tu veux dire quelque chose, X ? ». Donc simultanément le $\mathrm{M}$ se reconnaît comme destinataire de l'interpellation et accepte l'interruption du fil de son discours.

Je crois donc que dans une perspective pragmatique, il est possible de considérer le lever de doigt, parce qu'il est ritualisé et spécifiquement codifié en situation scolaire, comme un signe d'interpellation. Comme ce signe n'est pas langagier, nous ne pourrons pas l'analyser ici, mais il mérite d'être signalé également à cause de sa fréquence : dans les corpus vidéo que j'ai pu observer, le lever de doigt est la manière la plus fréquente pour les élèves d'interrompre l'enseignant. Il est infiniment plus fréquent, par exemple, que l'apostrophe en « maître », « maîtresse » ou l'usage du prénom de l'enseignant (4 à 5 fois plus de doigts levés que d'apostrophes). Son caractère rituel, dans la situation de classe, en fait un signe spécifique. 
Observons maintenant les formes langagières. Elles relèvent de trois types :

- des GN en apostrophe, et dans ce cas il s'agit presque toujours de noms propres, prénoms ou termes génériques « madame ", " maîtresse », « maître », etc.

- des interjections

- des injonctifs.

L'interjection la plus fréquente est «hé », déjà donnée par Demonnet (2006) comme marque d'interpellation dans les écrits contemporains. Elle se réalise simplement en « hé » ou légèrement modulé, « hep », ou répété « hé ! hé ! ». L’interjection est utilisée le plus souvent par l'enseignant, plus rarement par les élèves pour s'interpeller entre eux, mais pratiquement jamais (et dans ce cas-là, l'élève se fait reprendre) par un élève pour interpeller l'enseignant.

On trouve également en grand nombre "bon", classiquement considéré comme un marqueur discursif (Dostie \& Pusch, 2007) dans lequel Hansen (1998) a distingué des emplois discursifs et interjectifs. En situation de classe, «bon» paraît clairement interjectif quand il apparaît - c'est souvent le cas - en début de séance, manière pour l'enseignante d'interpeller l'ensemble des élèves pour les ramener au travail :

Bon alors on y va - les consignes sont au tableau je perds pas de temps

Les injonctifs observés sont "allez » à l'oral et «voyons! » à l'écrit. Sierra Soriano les classe comme desinterjections, au motif qu'elles «présentent une perte de contenu sémantique et un figement morpho-syntaxique au fur et à mesure qu'elles acquièrent une valeur émotive ou expressive" (Sierra-Soriano, 2006: 73). Et de fait, leur valeur est proche de celle des interjections commentées plus haut, même si la concurrence entre «allons » et "allez » rend tout de même difficile le classement de "allez » comme une interjection, dans la mesure où subsiste une variation flexionnelle. À l'oral, plus largement qu'une interruption de la parole, «allez» provoque une interruption d'activité, le plus souvent pour une mise en activité (mise au travail). À l'écrit, « voyons » inscrit en marge, comme un signal précédant un commentaire de l'écrit de l'élève, fonctionne bien comme un interrupteur de parole, ici la reconstitution par la lecture de la parole écrite. C'est au sein de l'écrit, interrompant de ce fait la linéarité de la lecture et l'acte d'énonciation que constitue toute lecture, que vient s'inscrire l'interpellation. Comme l'a montré Détrie (2007) à propos de l'apostrophe, il y a ici un changement de niveau énonciatif: l'échange auteur-lecteur, sur lequel est fondée la lecture du texte, devient soudain explicitement en un échange professeur-élève; même si les entités de l'élève et du professeur ne sont évidemment pas absentes lors de la simple lecture, dans laquelle communiquent un auteur et un lecteur, c'est la relation professeur-élève qui est rendue saillante par l'interpellation en marge. L'interpellation fait apparaitre les deux niveaux de lecture qui coexistent dans le pacte scolaire : un lecteur prend connaissance du texte d'un auteur et indissociablement un enseignant évalue un élève.

Les GN en apostrophe sont les formes d'interpellation les plus nombreuses à l'oral dans le cadre de l'interpellation d'un élève par un enseignant.

31 Syntaxiquement, le GN est généralement détaché en tête de paragraphe oral (terminologie de Morel), la suite étant le plus souvent un énoncé injonctif. La structure [énoncé injonctif $+\mathrm{GN}$ en apostrophe] (type «au travail, Damien! »), que nous avons considérée comme une adresse, représente $20 \%$ des occurrences associant un GN en apostrophe et un prédicat. 
Lorsque c'est un élève qui interrompt le discours du M par une apostrophe (« maître ! / «maîtresse!» / prénom de l'enseignant), les configurations sont les mêmes: GN interpellatif seul, ou bien suivi d'une prédication.

Il s'agit donc, comme on pouvait s'y attendre, de configurations d'apostrophe très courantes.

Plus rarement, elles peuvent être insérées dans le discours de l'enseignant et elles semblent fonctionner pragmatiquement en lien avec ce discours. Exemple: le M veut faire distribuer des documents, il va donc avoir recours à l'élève « de service » ce jour-là :

Bon- distributeur - Pierrick- tu distribues ça aux CE2 - on lit le document

Le premier mot, un nom sans déterminant, fonctionne comme une étiquette pré-posée au Npropre qui le suit. Il peut s'agir d'un marqueur d'étape, il a en tout cas une valeur métadiscursive qui pourrait se gloser en "dans la catégorie « distributeurs", j’appelle Pierrick». Le paragraphe "Pierrick! tu distribues ça aux CE2» constitue un îlot énonciatif adressé à un seul élève, tandis que le reste du discours s'adresse à tous, et constitue un commentaire de ce qui est en train de se passer (distribution- lecture).

Apparaît enfin un type d'énoncé difficile à classer a priori comme une interpellation mais qui en possède des fonctions pragmatiques. Il s'agit de commentaires de l'enseignant, qui paraît ne pas dévier syntaxiquement du fil de son discours, mais qui en fait s'adresse à des élèves.

37 Exemple :

quelquefois- quand on fait des sciences- j'aimerais bien que Baptiste se taise quelquefois- quand on fait des sciences- on sait déjà des choses vous savez déjà plein de choses

38 Il ne s'agit pas ici d'une interpellation au sens classique du terme: le paragraphe «j'aimerais bien que Baptiste se taise» fonctionne plutôt comme une incise. Mais l'intonation et le volume de la voix concourent à donner au paragraphe «j'aimerais que Baptiste se taise » une valeur pragmatique proche de celle qu'aurait une interpellation du type «Baptiste, tais toi !». D'autre part, la répétition de « quelquefois quand on fait des sciences », qui encadre l'incise, ne permet pas de considérer cet énoncé comme une partie non problématique du discours.

Il me parait difficile de classer cet énoncé comme une forme d'interpellation parce qu'il n'a aucune caractéristique des formes classiques, interjections et apostrophes. Néanmoins, du point de vue perlocutoire, il joue un rôle semblable à une interpellation. Cette difficulté à classer me parait refléter la nature linguistiquement hybride de l'interpellation, qui n'est pas une catégorie syntaxique ni lexicale mais qui n'est tout de même pas sans lien avec la syntaxe et le lexique. C'est un des intérêts de la situation didactique du point de vue de cet acte de langage très courant en classe, et qui se signifie de manière extrêmement variée, jusqu'à, nous l'avons vu, pouvoir n'être que gestuelle. Peut-être faut-il envisager, du point de vue des formes, une interpellation «au sens restreint ", qui réponde aux critères à la fois pragmatiques et lexico-syntaxiques de l'interpellation, tels qu'ils paraissent admis dans la littérature. Et lui adjoindre une interpellation «au sens large», en s'appuyant uniquement sur l'identification pragmatique, pour aller vers une définition en termes d'acte de langage. 


\section{Les fonctions de l'interpellation en situation de classe}

40 Je vais m'interroger ici sur le rôle de l'interpellation dans l'économie de la parole, et la distribution de la parole dans la classe. L'acception de l'interpellation comme « interruption de discours » va être élargie à la notion d'« interruption d'action » dans des cas spécifiques.

41 La situation didactique est, nous l'avons dit, une situation essentiellement discursive. L'interpellation s'y déroule dans trois types d'interactions :

Premier type, l'interpellation constitue une interruption de la parole magistrale : c'est le cas

- quand le discours du maître est interrompu par l'interpellation d'un élève, qu'elle prenne la forme d'une interpellation langagière ou d'un geste (le geste d'interpellation ritualisé en classe étant le lever de doigt) ;

- dans le cas d'auto-interruptions, qui sont en fait des réponses à des initiatives des élèves face auxquelles le maître doit réagir : il s'agit d'attitudes, de postures, de gestes inconvenants dans la situation, que le maître souhaite interrompre, et cette interruption constitue elle-même une interruption du discours magistral ; exemple d'un tel énoncé, l'enseignant s'adressant à un élève peu attentif : « X, écoute au lieu de t'amuser ».

Deuxième type, l'interpellation constitue une interruption par l'enseignant du discours et/ou de l'activité des élèves : c'est le cas

- lors de l'interpellation d'un élève par le maître après qu'il a posé une question, aucun élève ne se désignant pour répondre

- lors de l'interpellation d'un élève par le maître pour rompre le silence, dans un moment de travail individuel. Ce type d'interpellation, bien plus fréquent qu'on ne pourrait le croire, est sans doute imputable à une peur du silence maintes fois constatée chez les enseignants, en particulier les enseignants débutants, qui paradoxalement se montrent très soucieux d'obtenir le silence mais qui ne supportent pas longtemps un silence authentique, qu'ils rompent par des prises de parole sporadiques.

Ce deuxième type d'interpellation, quand le maître interpelle un ou des élève(s), joue certainement un rôle dans l'économie de la parole, réaffirmant le maître dans sa position surplombante et de contrôle. Les énoncés destinés à distribuer une parole implicitement demandée (par une question posée préalablement par exemple) se répartissent en deux formes de distribution à peu près égale : le prénom d'un élève seul / le prénom suivi d'un prédicat généralement de modalité interrogative. Les énoncés interrompant le silence lors d'une activité individuelle sont très massivement prédicatifs, avec une structure simple du type $\mathrm{X}, \mathrm{Y}$ où $\mathrm{X}$ est le prénom d'un élève (rarement plusieurs) et $\mathrm{Y}$, le prédicat.

Troisième type : en fond sonore et presque en continu, les interpellations d'élève à élève. Elles sont très fréquentes dans une classe, à tous les moments, et prennent des formes extrêmement variées : interjection seule, prénom seul, interjection ou prénom + prédicat, et l'ensemble des gestes et bruits divers permettant d'attirer l'attention. Finalement en situation didactique il est assez difficile d'envisager classiquement l'interpellation en termes binaires: $\mathrm{X}$ interpelle $\mathrm{Y}$. Je crois que l'on peut parler d'un 
«fond interpellatif » qui se manifeste de temps en temps, dans des interactions $\mathrm{M} / \mathrm{E}, \mathrm{E} / \mathrm{M}$ ou E/E. ${ }^{4}$

Si l'on prend ces interactions comme un ensemble, on peut considérer l'existence d'une « parole collective » qui serait celle de la classe, maître et élève confondus. Une " parole collective » où ont également place les silences, les pauses. Il devient possible d'envisager les interpellations, d'où qu'elles viennent, comme des ponctuations de cette parole collective, des sortes de marqueurs prosodiques à un niveau macro-discursif. La "parole collective » d'une classe ${ }^{5}$ est presque toujours faite d'échanges: le cours magistral n'est plus de mise à l'école primaire, et la prise de parole des élèves est fréquente. Elle se fait dans un cadre langagier spécifique: l'école, où les individus prennent la parole en s'inscrivant dans leurs statuts respectifs d'apprenant et d'enseignant. Le modus de cette parole est souvent extrêmement sobre, cette sobriété reflétant peut-être l'idée que ce qui compte, c'est le dictum. Cette parole scolaire a en outre la spécificité d'être extrêmement contrôlée, à la fois dans ses contenus et dans ses formes : à l'école il s'agit d'énoncer un discours dans une langue correcte.

En décalage avec ces échanges à finalité instructive, les interpellations

- sont des énoncés principalement descriptibles en termes de modus

- donnent la parole à des entités énonciatives qui ne sont pas centralement l'enseignant et l'apprenant mais plutôt, me semble-t-il, le maître et l'élève, ou l'adulte et l'enfant, en tout cas je crois que les interpellations privilégient l'expression des individus plus que celle du statut que leur confère l'école

- sont des énoncés proférés dans des situations empreintes d'émotion, et à ce titre paraissent échapper partiellement au contrôle méta-énonciatif, ou pour le dire autrement paraissent plus spontanées que des énoncés plus nettement scolaires.

C'est donc une strate énonciative peu apparente en classe que donnent à voir les interpellations. Pour en étudier l'ensemble des modalités, il faut sans doute quitter le niveau strictement linguistique pour s'attacher aussi aux gestes de l'interpellation, dont j'espère avoir montré que certains avaient, à l'école, valeur de signes. ${ }^{6}$

\section{Pour clore sans conclure}

Ces considérations, qui visaient à débrouiller un peu le terrain fort accidenté de l'analyse des interactions en classe et spécifiquement de l'interpellation, courent le risque de l'avoir embrouillé davantage. Il me semble que l'interpellation partage avec l'apostrophe la mise en évidence d'un changement de pallier énonciatif, descriptible en termes d'interlocution et d'acte de langage; elle me paraît partager avec l'interjection le caractère relativement peu contrôlé de l'énonciation, et ceci même dans la nomination, comme cela est apparu avec l'exemple de la langue étrangère. Son caractère hybride, qui s'accorde mal avec les catégories linguistiques habituelles, vient rappeler l'hétérogénéité des classements et les hésitations qui en découlent dès lors qu'on s'intéresse à l'énonciation en acte. Ce essai de débroussaillage, qui n'a prétention d'être ni exhaustif, ni définitif, constituera, je l'espère, une propédeutique à des travaux futurs qui viendront étayer, prolonger, refonder les quelques bases que s'efforce de poser ce volume. 


\section{BIBLIOGRAPHIE}

Berthoud, A.-C. (1996) Paroles à propos. Approche énonciative et interactive du topic. Paris : Ophrys.

Charaudeau, P. (1992) Grammaire du sens et de l'expression. Paris : Hachette éducation.

Demonet, M.-L. (2006) « Eh/hé : l'oralité simulée à la renaissance ». Langages, n¹61. 57-72.

Détrie, C. (2006) De la non-personne à la personne : l'apostrophe nominale. Paris : CNRS.

Develay, M. (2007) Donner du sens à l'école. ESF.

Dostie, G. \& Pusch C. D. (2007) (dir) Les Marqueurs discursifs. Langue Française, nº 154.

Hansen, R. (1998). The Socialization of Technology Teachers: Two Unique Cases. Journal of Industrial Teacher Education, 35(2), 29-41.

Morel, M.A. \& Bouvet, D. (2001) « Les réalisations formelles de la co-énonciation : cooccurence et distribution des indices sur 3 plans : morphosyntaxique, intonatif, posturomimogestuel. » In Cave et al. Oralité et gestualité. Paris : L'Harmattan. 482-487.

Morel, M.-A. (2007) « Le postrhème dans le dialogue oral en français ». L'Information grammaticale, $\mathrm{n}^{\circ} 113.40-45$.

Noailly, M. (1995) (dir.) Noms propres et nomination. Paris : Klincksieck.

Schegloff, E.A. (1980) `Preliminaries to Preliminaries: "Can I Ask You a Question?"', Sociological Inquiry 50: 104-52

Sensevy, G \& Mercier, A (2008) (dir.) Agir ensemble. L'action didactique conjointe du professeur et des élèves. Rennes : PUR.

Sierra-Soriano, A. (2006) Interjections issues d'un verbe de mouvement : étude comparée français-espagnol. Langages, $\mathrm{n}^{\circ} 161.73-90$

\section{NOTES}

1. Alors me paraît ici avoir les deux valeurs possibles, les substitutions possibles renforçant cette interprétation (il est facile de substituer à alors : eh bien, hé, etc. Toutefois l'absence de pause marquée laisse émettre des réserves. C'est la prosodie qui serait déterminante ici. Comme ce n'est pas le propos principal je ne m'y étendrai pas.

2. En revanche, au travail - eh est parfaitement admissible. Voir à ce propos Demonnet 2006.

3. Le même constat est fait dans le cas de phrases interrogatives: on met lecture ou pas maîtresse ? / *on met lecture ou pas hé ?

4. Sans parler de l'interpellation $M / M$, qui est une sorte d'interpellation intérieure, donc non manifestée, que nous ne pouvons pas observer, mais qui apparaît quelquefois dans les discours magistraux que l'on peut recueillir par exemple dans les auto-confrontations des enseignants avec un film tourné dans leur classe : «là je me suis dit: hé ho, ça va pas, tu dérailles complètement! Et c'est pour ça que j'ai réorienté après, ils ne comprenaient rien en fait ». Ici nous restons à l'observable attesté in vivo, donc à des interactions $\mathrm{M} / \mathrm{E}, \mathrm{E} / \mathrm{M}$ ou $\mathrm{E} / \mathrm{E}$. 
5. Pour une théorisation de ces notions en didactique, cf. la théorie de l'action conjointe développée par Sensevy \& Mercier (2008).

6. Gestuelle que certains linguistes de l'oral, je pense par exemple à Marie-Annick Morel et Danielle Bouvet (2001), intègrent aujourd'hui à leurs analyses.

\section{AUTEUR}

CLAIRE DOQUET-LACOSTE

ITEM, UMR 8132 \& Cread, EA 3875 